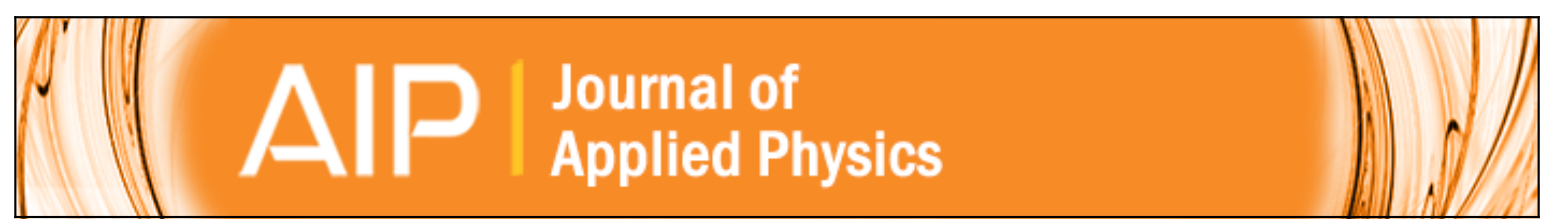

\title{
Dynamical scaling in fragmentation
}

Kaline Coutinho, Sadhan K. Adhikari, and M. A. F. Gomes

Citation: Journal of Applied Physics 74, 7577 (1993); doi: 10.1063/1.354984

View online: http://dx.doi.org/10.1063/1.354984

View Table of Contents: http://scitation.aip.org/content/aip/journal/jap/74/12?ver=pdfcov

Published by the AIP Publishing

\section{$\underset{\substack{\text { Aultoring } \\ \text { sing }}}{A}$ Re-register for Table of Content Alerts}




\title{
Dynamical scaling in fragmentation
}

\author{
Kaline Coutinho \\ Departamento de Física, Universidade Federal de Pernambuco, 50739 Recife, Pernambuco, Brazil
}

Sadhan K. Adhikari

Instituto de Fisica Teórica, Universidade Estadual Paulista, 01405 São Paulo, São Paulo, Brazil

M. A. F. Gomes

Departamento de Física, Universidade Federal de Pernambuco, 50739 Recife, Pernambuco, Brazil

(Received 29 June 1993; accepted for publication 2 September 1993)

The dynamics of a fragmentation model is examined from the point of view of numerical simulation and rate equations. The model includes effects of temperature. The number $n(s, t)$ of fragments of size $s$ at time $t$ is obtained and is found to obey the scaling form $n(s, t)$ $\sim s^{-\tau} t^{u s^{\gamma}} e^{-\rho t} f\left(s / t^{2}\right)$ where $f(x)$ is a crossover function satisfying $f(x) \simeq 1$ for $x \ll 1$ and $f(x) \ll 1$ for $x \gg 1$. The dependence of the critical exponents $\tau, w, \gamma$, and $z$ on space dimensionality $d$ is studied from $d=1$ to 5 . The result of the dynamics on fractal and nonfractal objects as well as on square and triangular lattices is also examined.

\section{INTRODUCTION}

Fragmentation of objects occurs in a variety of physical and chemical phenomena in nature, which include explosion, fracture, grinding and crushing of solids, such as rocks and minerals, ${ }^{1}$ droplet breakup, ${ }^{2}$ chemical or radioactive consumption, ${ }^{3}$ polymer degradation, ${ }^{4}$ nuclear fragmentation, ${ }^{5}$ among many others. All these fragmentation processes can be broadly classified into two categories: (i) those that conserve mass, e.g., grinding and crushing of rocks, ${ }^{6}$ and (ii) those that consume mass, e.g., the chemical dissolution of an object by a reactive agent. ${ }^{3,7-9}$ Though the phenomena of fragmentation is of interest in a host of areas, the present understanding of the dynamics associated with these nonequilibrium fragmentation processes is very limited compared to other equilibrium phenomena. The scope of the present work is to shed light on the dynamics of breakage, or fragmentation, using numerical simulations under a variety of situations.

The term fragmentation is usually referred to as any process that reduces the connectivity of an object and thus generates smaller objects known as fragments. However, in the present work, we would like to restrict ourselves to fragmentation processes with a physical motivation.

Formation of large, random clusters by nonequilibrium processes is common to a class of physical and chemical phenomena in nature which include (a) fragmentation processes, ${ }^{1,3,6-9}$ (b) aggregation processes, ${ }^{10}$ and (c) an appropriate mixture of aggregation and disaggregation ${ }^{11}$ among others. Most of the theoretical studies of these processes have concentrated on a microscopic evaluation of the cluster size distribution $n(s, t)$, the number of fragments of size $s$ at time $t$, and of the total number of frag. ments $N(t)$ at time $t$. A simple dynamic scaling for $n(s, t)$ of the form

$$
n(s, t) \sim s^{-\tau} t^{-w} f\left(s / t^{z}\right),
$$

was first observed in cluster-cluster aggregation, ${ }^{10}$ where the cutoff function $f(x) \simeq 1$ for $x \ll 1$ and $f(x) \ll 1$ for $x \gg 1$. The interesting feature of Eq. (1.1) is a power law decay in both $t$ and $s$ for large $t$ and small $s$. Later other studies have confirmed scaling (1.1).

It is interesting to ask whether scaling (1.1) has only limited validity or is valid in other nonequilibrium processes dealing with cluster size distribution. Here we study the behavior of $n(s, t)$ in three different fragmentation models: corrosive rain, random walk, and alternate horizontal and vertical lines models. The corrosive rain model is a realistic fragmentation model and can describe the fragmentation of a solid produced by oxidation or dissolution. The other two models perhaps simulate fragmentation under stress and under some other conditions. ${ }^{1,4,12,13}$ These two other models are studied in order to investigate the model dependence of the results found with the corrosive rain model. The variance with respect to dimensionality, finite size, and geometric properties of the fragmented solid is also studied.

Initially, we studied in detail a particular case of the corrosive rain model at infinite temperature $(T=\infty)$. In this case, the statistical thermal fluctuations are greater than any energy variations caused by the process of fragmentation. Therefore we call it the random domain. In this domain the equations for some variables, e.g., mass, $M(t): d M(t) / d t=-M(t)$, can be easily solved. However others variables, such as, $n(s, t)$, do not have a trivial behavior during the process.

We have studied fragmentation processes through numerical simulation and dynamical probabilistic rate equations. Specifically, we studied the quantities, $n(s, t), N(t)$, the total mass $M(t)=\Sigma_{s} n(s, t) s, \bar{s}(t) \equiv M(t) / N(t)$, average coordination $\bar{q}(t)$, and the diversity of fragments $D(t) .{ }^{9}$ The diversity $D(t)$ is the number of different mass fragments (irrespective of shape) present at time $t$. We tried to find out solutions to dynamical probabilistic rate equations, whenever possible, under simplifying assumptions and compared these solutions to predictions of numerical simulation.

In our numerical simulation of fragmentation we find 
that the $s$ dependence of $n(s, t)$ is always given by (1.1), e.g., $n(s, t) \sim s^{-\tau} f\left(s / t^{z}\right)$. However, we find a distinct $t$ dependence of $n(s, t)$. Explicitly, we find the following general distribution in our numerical simulation of fragmentation processes

$$
n(s, t) \sim s^{-\tau} t^{w s^{\gamma}} e^{-\rho t} f\left(s / t^{z}\right)
$$

where $\tau, w, \gamma$, and $z$ are exponents that satisfy certain conditions, and $\rho$ is a constant that controls the exponential consumption of mass for large $t$. It is found that $w=(\tau$ $-2) z$ for $\gamma=0$, and that the exponent $\gamma$ exhibits a rapid variation when the initial coordination of the object, $\bar{q}_{0}$, is near 3: for $\bar{q}_{0}<2.5, \gamma=0$ and for $\bar{q}_{0} \geqslant 3.2, \gamma=0.28 \pm 0.03$. By coordination we mean the number of neighbors or chemical bonds of an occupied site. Scaling (1.2) also governs the $t$ dependence of the total number of fragments $N(t)$. Hence we are led to conclude that the $s$ dependence of $\mathrm{Eq}$. (1.1) is more universal, while the $t$ dependence is not. Scaling (1.2) is valid in fragmentation models considered by us and is distinct from scaling (1.1). First, the exponent $w$ has changed sign. Second, in Eq. (1.1) the cutoff function $f$ destroys the algebraic scaling in $t$ for small $t$, whereas because of the exponential function in Eq. (1.2) the algebraic scaling in $t$ is destroyed for large $t$. Consequently, in Eq. (1.2) $n(s, t)$ [and also $N(t)$ ] exhibits a power law increase in $t$ for small $t$ and an exponential decay for large $t$. In Eq. (1.1) $n(s, t)$ first increases to a maximum and then exhibits a power law decay in $t$ for large $t$. Though we have studied the $s$ dependence of $n(s, t)$ in our models, we are mostly concerned with the results for the $t$ dependence of $n(s, t)$ and $N(t)$ as they are distinct from scaling (1.1). In Sec. IV A we present a discussion of scaling (1.2), especially its $t$ dependence.

The present scaling (1.2) should be considered as a generalization of results of Gomes and Vasconcelos ${ }^{9}$ and Sahimi and Tsotsis ${ }^{8}$ to include large intervals of $t$ and some other models of fragmentation. However, the appearance of a power law in an exponent in scaling (1.2) is new to numerical simulations of not only fragmentation models but also aggregation models. But such scaling is not uncommon in other areas of physics. Dynamics involving disordered systems frequently exhibit power laws in an exponent. For example, much attention has been focused recently on relaxation phenomena in complex condensed matter systems where correlation functions decay as $\phi(t) \sim \exp \left(-w t^{\gamma}\right)$, with $0<\gamma<1$, or $\phi(t) \sim t^{-w(\ln t)^{\gamma}} \cdot{ }^{14}$

In addition to studying the cluster size distribution in fragmentation we also studied certain macroscopic variables of fragmentation processes to seek universal scaling relations among them. We believe that these macroscopic properties, unlike the cluster size distribution, will be governed by some general characteristics of the dynamics rather than its full intricate complexities.

In particular we studied two quantities during the fragmentation processes: the total fragment number $N(t)$, and the diversity of fragments $D(t)$. For several kinds of fractal and nonfractal objects and attacks in space dimensions $d=1-5$ we find as a result of numerical simulation that

$$
\mathscr{N} \sim M_{0} \sqrt{q_{0}}
$$

and

$$
\mathscr{N} \sim \mathscr{D}^{2}
$$

where $\mathscr{N}$ is the maximum of $N(t)$ and $\mathscr{D}$ is the maximum of $D(t)$, and $M_{0}$ is the initial mass and $\bar{q}_{0}$ is the average initial coordination of the object. Essentially, the same scaling properties are established using nondynamical arguments independent of space dimension, and using analytic dynamical rate equations under some simplifying assumptions.

The present scalings (1.2) and (1.4) were first observed in studies of fragmentation in the random domain of the corrosive rain model. In the random domain all particles on a lattice when attacked by an external agent could be removed with equal probability. This is true when the statistical thermal energy of the particles is much higher than their binding energies, which is the actual state of affairs at infinite temperature. In order to take the real significance of our results, we compare them with those at finite temperature, known as the kinetic domain. In this case the particles near the boundary of the object, which are loosely bound to the object are more vulnerable to removal. In this domain there is a competition between the thermal energy and the energy variation caused by the fragmentation process in the corrosive rain model. Therefore, we call it the kinetic domain.

In our numerical simulations at finite temperature of the corrosive rain model (kinetic domain), we observe the same scalings (1.2) and (1.4) found in the random domain, and the numerical values of the exponents $\tau, w, \gamma$, and $z$ remain unchanged. In order to test the generality of these exponents, we also compare it with experimental ${ }^{15,16}$ and theoretical ${ }^{9,10,17}$ exponents. Certain quantities, such as, the total mass of the object $M(t)$, have the trivial exponential behavior in the random domain. It is relevant to point out that the quantities governed by scaling relations (1.2) and (1.4), which are the principal results of this work, maintain the power law behavior in the random domain.

Another interesting observation is that the relationship $\mathscr{N} \sim \mathscr{D}^{2}$ is valid both in the random and the kinetic domain of the rain model and in two other models studied (random walk model and alternate lines model). Though scaling relations have been observed in various nonequilibrium phenomena the present scalings (1.3) and (1.4) involving $\mathscr{N}$ and $\mathscr{D}$ are unique in yielding the same exponent for different space dimensions, lattice symmetry, and various types of attacks. In contrast to other scalings the robustness of the present scaling is worth emphasizing.

In Sec. II we present the models of fragmentation which we use in numerical simulation. In Sec. III we develop the probabilistic rate equations for various quantities in the three models of fragmentation and find out solutions to some of these equations under simplifying assumptions. In Sec. IV we present results of numerical simulations in the present models of fragmentation and compare them, 
wherever possible, to the analytic solutions of the rate equations. Finally, in Sec. V we present a brief discussion of our findings.

\section{THE MODELS}

In this work we shall employ three attack algorithms for fragmentation: (i) fragmentation by corrosive rain, (ii) fragmentation by random walk, and (iii) fragmentation by cutting the object by alternate horizontal and vertical segments.

First, we propose to model the attack of an object by a kind of corrosive rain. The units that form this rain may be considered as molecules of a chemical reagent in some corrosion process. ${ }^{9}$ In the numerical simulation of fragmentation by corrosive rain with mass consumption we use the following algorithm. An attack is made on site $i$ of an object, defined on a lattice of size $L$, chosen at random. The reactivity (or probability of successful attack) at a lattice site $i$ of the solid is taken to be proportional to the Boltzmann factor $\exp \left(-E_{i} / k T\right)$, with $E_{i}=q_{i} E$, where the coordination $q_{i}$ refer to site $i, E$ is some characteristic energy of each (chemical) bond, $k$ is the Boltzmann constant, and $T$ is the temperature. Here, $E_{t}$ represents the energy to break $q_{i}$ chemical bonds of site $i$, each of energy $E$ at temperature $T$. With this reactivity, the sites that are weakly connected to the system, e.g., the ones with low $q_{i}$ are more vulnerable to the attack. The Boltzmann factor is particularly interesting to control the attack or the reactivity at site $i$ in view of its relationship with many situations in chemical kinetics. For a finite $T$ one is in the kinetic domain where the rate of consumption is controlled by the rate of reaction. For an infinite $T$ one has the random domain where the rate of consumption is controlled only by the randomness of the selection of sites.

If site $i$ is unoccupied, it continues to be unoccupied. If it is occupied, it is unoccupied with probability $p\left(q_{i}, \Theta\right)=\exp \left(-q_{i} / \Theta\right)$, where $\Theta(\equiv k T / E)$ is the reduced temperature. With this reactivity the sites that are weakly connected to the system are more vulnerable to the attack at a finite temperature. The above procedure is repeated until all sites are unoccupied. This model simulates the fragmentation of a solid (with mass consumption) attacked by a chemical reagent. This model of attack is more physical in one and two space dimensions. The results for higher dimensions confirm the universality of the scaling relations.

In the process of fragmentation by random walk the only difference from the above process of fragmentation by corrosive rain is in the way the site $i$ is chosen. First, an attack is made on a site $i$ of the object chosen at random. If site $i$ is unoccupied, it continues to be unoccupied. If it is occupied, it is unoccupied with probability $p\left(q_{i}, \Theta\right)$. Next an attack is made on any of the nearest neighbors of site $i$ chosen with equal probability. This procedure is repeated until all sites are unoccupied. This random walk model is subject to the periodic boundary conditions. Although, this model is not related in an obvious way to a specific physical system, there are considerations which have led us to examine its properties. The simple rules of this model are reminiscent of dynamic processes of failure in brittle materials. These materials fragment when cracks appear, grow, and propagate as a result of dynamical processes, such as in rock blasting. ${ }^{12}$ If the solid is homogencous the first crack will propagate unstably and lead to complete fracture. ${ }^{13}$ Fragments are formed when the crack density is sufficiently high so as to fully surround pieces of matter. Also, in two dimensions this model reminds the walk of an ant which cuts a leaf as it walks on the surface of the leaf. In the present model the random walker simulates the propagation of crack or of the ant.

In the process of the fragmentation by cutting with alternate horizontal and vertical lines in two space dimensions, without mass consumption, we use the following algorithm. In this case the attack does not consume mass but only breaks certain links or (chemical) bonds between tiny objects, such as molecules, arranged on a lattice. In a two-dimensional fragment a site $i$ which has the horizontal link to the left intact is chosen at random. Then this link and other $\left(n_{i}-1\right)$ parallel horizontal links needed to break the fragment in two parts are broken with probability $p\left(n_{i}, \Theta\right)=\exp \left(-n_{i} / \Theta\right)$ where $\Theta(\equiv k T / E)$ is again the reduced temperature, here $n_{i}$ links specify the size of the fracture. Next a site $i$ with an upward vertical link intact is 'chosen at random. Then this vertical link and other $\left(n_{i}-1\right)$ parallel vertical links needed to break the fragment in two parts are broken with probability $p\left(n_{i}, \Theta\right)$ so that the fragment is broken into two pieces. Such horizontal and vertical fractures are repeated alternately until all the links are broken. This model simulates the fragmentation of a solid under mechanical stress. In contrast to the first two models of fragmentation, where there is consumption of mass, this model conserves mass. This fragmentation model is a generalization of a model considered and studied in detail by Mott and Linfoot in $1943,{ }^{18}$ and also exhaustively discussed recently by Grady and Kipp. ${ }^{6}$ The Mott-Linfoot model corresponds to infinite temperature and can be solved analytically.

\section{PROBABILISTIC RATE EQUATIONS}

Before presenting results of actual numerical simulation of fragmentation, we present in this section probabilistic rate equations for time evolution of some macroscopic quantities. There has been studies of fragmentation using rate equations for $n(s, t)$ by various authors in diverse contexts. ${ }^{19,20}$ Instead of presenting general rate equations for $n(s, t)$ we develop simplified rate equations for certain quantities studied in our numerical simulation. Such simplified rate equations allow analytic solutions under some restrictions, which will allow us to compare the results of numerical simulations with those of the rate equations. Usually, the general rate equations presented recently ${ }^{19,20}$ are quite involved and do not permit analytic solutions to be compared with numerical simulation. Also, in the present work we are mainly interested in processes involving mass loss. They are essential in chemical kinetics, e.g., during oxidation and dissolution of solids. Most of the recent analytical studies on fragmentation ${ }^{19,20}$ were limited to processes where total mass is conserved. 
In particular, we derive rate equations for the number of fragments (zero order moment), $N(t)=\Sigma_{s} n(s, t)$, mass (first order moment), $M(t)=\Sigma_{s} n(s, t) s=\Sigma_{q_{i}} m\left(q_{i}, t\right)$, average fragment size, $\bar{s}(t)=M(t) / N(t)$, and average coordination, $\bar{q}(t)=\Sigma_{q_{i}} m\left(q_{i}, t\right) q_{i} / M(t)$, where $m\left(q_{i}, t\right)$ is the number of occupied sites at time $t$ with coordination $q_{i}$.

In the present work, every time an attack is made on a site the time $t$ is increased by $\Delta t=1 / \Omega_{0}$, where $\Omega_{0}$ is the total number of sites-occupied and unoccupied-of the lattice available for the attack. Hence in unit time all the sites of the lattice may be visited, independent of the size or the topology of the object, or the dimension of the space employed. Also at about $t=10$ the whole mass is consumed independent of initial size, connectivity, or space dimensionality of the object. In this time millions or even trillions of attacks could be made on the object, but the variation of time in the present units remain of the order of 10.

\section{A. Fragmentation by corrosive rain}

In this case the rate equation for the variation of mass could be written as

$$
\begin{aligned}
\frac{d}{d t} M(t)= & \sum_{q_{i}}\left[\frac{m\left(q_{i}, t\right)}{\Omega_{0}}\right] p\left(q_{i}, \Theta\right) \\
& \times\left[\frac{M\left(t+1 / \Omega_{0}\right)-M(t)}{1 / \Omega_{0}}\right],
\end{aligned}
$$

where the quantity in the first square bracket on the right hand side is the probability to visit an occupied site of coordination $q_{i}$, and the quantity $p\left(q_{i}, \Theta\right)$ represents the probability of unoccupying this site. The quantity in the last square bracket denotes the variation of mass as a result of this attack. Finally, this expression is summed over coordination $q_{i}$ in order to include all possible attacks. Now noting that $M\left(t+1 / \Omega_{0}\right)=[M(t)-1]$, this equation simplifies to

$$
\frac{d}{d t} M(t)=-\sum_{q_{i}} m\left(q_{i}, t\right) p\left(q_{i}, \Theta\right)
$$

Similarly, one can write the following equation that determines the time evolution of the average coordination, $\bar{q}(t)$, for all occupied sites of the solid

$$
\frac{d}{d t}[M(t) \bar{q}(t)]=\sum_{q_{i}} m\left(q_{i}, t\right) p\left(q_{i}, \Theta\right)\left[-2 q_{i}\right]
$$

where the term in the square bracket on the right-hand side of this equation is the variation of $M(t) \bar{q}(t)$ after successfully unoccupying site $i$. The first two terms on the righthand side of Eq. (3.3) are written in analogy to Eq. (3.1). Equations (3.2) and (3.3) provides $d \bar{q}(t) / d t$.

The rate equations presented in the last two paragraphs determine the time evolution of $M(t)$ and $\bar{q}(t)$. However, they can not be easily handled except in the special case of infinite reduced temperature, $\theta=\infty$, and we consider this special case in the following. In this case the fragmentation is random in nature, and the rate of con- sumption is only determined by the randomness in choosing a site, as the probability of consumption is unity in this case.

At infinite temperature $p\left(q_{i}, \Theta\right)=1$ and noting that $\Sigma_{q_{i}} m\left(q_{i}, t\right)=M(t)$ Eq. (3.2) becomes

$$
\frac{d}{d t} M(t)=-M(t)
$$

which yields the simple solution

$$
M(t)=M_{0} \exp (-t),
$$

with $M_{0}$ the initial mass. Under same conditions Eq. (3.3) becomes

$$
\frac{d}{d t}[M(t) \bar{q}(t)]=-2 M(t) \bar{q}(t) .
$$

Equations (3.4) and (3.6) easily lead to

$$
\frac{d}{d t} \bar{q}(t)=-\bar{q}(t),
$$

which yields the simple solution

$$
\bar{q}(t)=\bar{q}_{0} \exp (-t),
$$

with $\bar{q}_{0}$ the initial average coordination. Equations (3.5) and (3.8) are the principal results in this case, which will be verified in actual numerical simulation.

\section{B. Fragmentation by random walk}

In special case of infinite reduced temperature, $\theta=\infty$, the rate equations of the random walk model are very similar to the equations of the last subsection. In this case in Eq. (3.1) only the quantity in the first square bracket on the right hand side representing the probability to visit an occupied site has to be modified to the probability to find an occupied neighbor by the random walker. If the fully occupied lattice has coordination $Z$ for any site $i$, then the random walker has access only to $(Z-1)$ occupied sites of the $Z$ available neighbors, because one neighbor, the last step, must be unoccupied. Hence the probability to find an occupied neighbor by the random walker will be [ $Z$ $-1) / Z] \Sigma_{q_{i}}\left[m\left(q_{i}, t\right) / \Omega_{0}\right]$, since $p\left(q_{i}, \Theta\right)=1$. Consequently, Eqs. (3.2) and (3.3) should be modified in this case by the introduction of the factor $(Z-1) / Z$ on the right-hand side. Explicitly, these equations become

$$
\frac{d}{d t} M(t)=-\frac{(Z-1)}{Z} \sum_{q_{i}} m\left(q_{i}, t\right)
$$

and

$$
\frac{d}{d t}[M(t) \bar{q}(t)]=\frac{(Z-1)}{Z} \cdot \sum_{q_{i}} m\left(q_{i}, t\right)\left[-2 q_{i}\right] .
$$

The solutions for two equations above could be easily obtained to lead to the following solutions: $M(t)=M_{0} \exp (-c t)$ and $\bar{q}(t)=\bar{q}_{0} \exp (-c t)$, where $c=(Z-1) / Z$.

Hence the sets of rate equations for corrosive rain and random walk, at infinite reduced temperature, differ only 
by a multiplicative constant which depends on the dimension of the space and lattice symmetry. Note that in both cases one has the following simple relation

$$
\frac{M(t)}{\bar{q}(t)}=\frac{M_{0}}{\bar{q}_{0}} .
$$

\section{Fragmentation by alternate horizontal and vertical lines}

In this case the actual mass is conserved and it is interesting to introduce an active mass $\mathscr{M}(t) \equiv \Sigma_{n} m\left(n_{i}, t\right)$, where $\mathscr{H}(t)$ represents number of sites vulnerable to attack, e.g., the number of sites which has either the horizontal link to the left or the upward vertical link intact and $m\left(n_{i}, t\right)$ represents the number of sites vulnerable to attack which requires breaking $n_{i}$ links in order to generate a new fragment. The rate equation in this case can be written as

$$
\begin{aligned}
\frac{d}{d t} \mathscr{M}(t)= & \sum_{n_{i}}\left[\frac{m\left(n_{i}, t\right)}{\mathscr{M}(t)}\right] p\left(n_{i}, \Theta\right) \\
& \times\left[\frac{\mathscr{M}\left(t+1 / \Omega_{0}\right)-\mathscr{M}(t)}{1 / \Omega_{0}}\right] .
\end{aligned}
$$

The quantity in the first square bracket in this equation is the probability to visit an active site and the factor $p\left(n_{i}, \Theta\right)$ represents the probability to break $n_{i}$ links and generate a new fragment. The quantity in the last square bracket in this equation represents the variation in active mass $\mathscr{M}$ due to this attack. Finally, one has to sum this expression over all possible $n_{i}$ to find the total variation. Recalling that $\mathscr{M}\left(t+1 / \Omega_{0}\right)=\left[\mathscr{M}(t)-n_{i}\right]$ Eq. (3.12) readily simplifies to

$$
\frac{d}{d t} \mathscr{H}(t)=-\sum_{n_{i}} \frac{m\left(n_{i}, t\right)}{\mathscr{M}(t)} p\left(n_{i}, \Theta\right) n_{i} \Omega_{0} .
$$

In this case it is interesting to write the following rate equation for total fragment number $N(t)$ following the same reasoning used to write Eq. (3.12):

$$
\frac{d}{d t} N(t)=\sum_{n_{i}} \frac{m\left(n_{i}, t\right)}{\mathscr{M}(t)} p\left(n_{i}, \Theta\right)\left(\frac{N\left(t+1 / \Omega_{0}\right)-N(t)}{1 / \Omega_{0}}\right) .
$$

Noting that $N\left(t+1 / \Omega_{0}\right)-N(t)=1$ Eq. (3.14) simplifies to

$$
\frac{d}{d t} N(t)=\sum_{n_{i}} \frac{m\left(n_{i}, t\right)}{\mathscr{M}(t)} p\left(n_{i}, \Theta\right) \Omega_{0} .
$$

Equations (3.13) and (3.15) determines the rate of variation of active mass $\mathscr{M}(t)$ and number of fragments $N(t)$ in this case.

In the infinite temperature limit Eq. (3.13) for active mass does not simplify enough to be analytically tractable. But, recalling that $\Sigma_{n_{i}} m\left(n_{i}, t\right)=\mathscr{M}(t)$, and $p\left(n_{i}, \Theta\right)=1$ in the infinite temperature limit, Eq. (3.15) for total number of fragments, $N(t)$, reduces to

$$
\frac{d}{d t} N(t)=\Omega_{0}
$$

which has the simple solution

$$
N(t)=1+\Omega_{0} t .
$$

\section{NUMERICAL SIMULATION}

We used in numerical simulation various compact (nonfractal) objects on linear $(d=1$, and $\left.1.997 \leqslant \bar{q}_{0} \leqslant 2.000\right)$, square $\left(d=2\right.$, and $\left.3.85 \leqslant \bar{q}_{0} \leqslant 4.00\right)$, triangular $\left(d=2\right.$, and $\left.5.71 \leqslant \bar{q}_{0} \leqslant 6.00\right)$, cubic $(d=3$, and $\left.5.40 \leqslant \bar{q}_{0} \leqslant 5.94\right)$, and hypercubic $\left(d=4\right.$, and $7.20 \leqslant \bar{q}_{0} \leqslant 7.77$; $d=5$, and $8.75 \leqslant \bar{q}_{0} \leqslant 9.57$ ) lattices. Among porous (fractal) objects we used Sierpinski carpets $(d=2$, and $\left.3.03 \leqslant \bar{q}_{0} \leqslant 3.20\right)$, percolation clusters $(d=2$ and $\left.2.45 \leqslant \bar{q}_{0} \leqslant 2.47\right)$, and Menger sponges $(d=3$, and $\left.3.36 \leqslant \bar{q}_{0} \leqslant 3.96\right)$. For every objects we made an average over fifty simulations. We employed different objects in space dimension $d$ up to $5, \bar{q}_{0}$ up to almost 10 , and $M_{0}$ up to 6.4 million.

We made numcrical simulation employing three models of fragmentations described in Sec. III for both finite and infinite temperatures. The infinite temperature domain is termed the random domain as the probability of fragmentation is unity and independent of details. This domain deserves special attention as in this case we have some analytic results of rate equations to be compared to the results of numerical simulation. The finite temperature domain is termed the kinetic domain as the probability of fragmentation depends on physical details, such as, temperature, strength of chemical bonds, etc. In order to test the universality of our results we also considered finite temperature domain in present numerical simulation.

\section{A. Random domain: Infinite temperature}

First, we consider fragmentation by corrosive rain in the infinite temperature limit. This model of attack simulates an acid rain that attacks a surface. With time both the total number of fragments $N(t)$ and diversity $D(t)$ first increase till diversity reaches a maximum value $\mathscr{D}$ at time $t_{\mathscr{D}}$. With further attack $N(t)$ continues to increase with large fragments giving birth to many small fragments of same mass/size, but diversity starts to decrease. Finally, one encounters a very large number of small fragments, and $N(t)$ attains its maximum value $\mathscr{N}$ at time $t_{\mathscr{N}}$. These features of $N(t)$ and $D(t)$ are very universal and are maintained for fractal and nonfractal objects in all space dimensions and for a general class of attack. A typical plot of $N(t)$ and $D(t)$ vs $t$ is exhibited in Fig. 1.

In this case using the results of rate equations we first make certain previsions for the critical quantities $\mathscr{N}, t_{\mathscr{N}}$, $\mathscr{D}$, and $t_{\mathscr{D}}$. Later we shall present the study on the time evolution of the quantities. Using the definition, $M(t)$ $=N(t) \bar{s}(t)$, and Eq. (3.11) we have the relation

$$
N(t)=\frac{M_{0}}{\bar{q}_{0}} \frac{\bar{q}(t)}{\bar{s}(t)} .
$$

At the maximum of $N(l)$, where the fragments are small in size and large in number, the average coordination $\bar{q}\left(t_{\mathscr{N}}\right)$ is related to $\bar{s}\left(t_{\mathscr{r}}\right)$ purely by geometric arguments. For example, for $\bar{s} \leqslant 2, \bar{q}=\bar{s}-1$; for larger $\bar{s}$ the relation between 


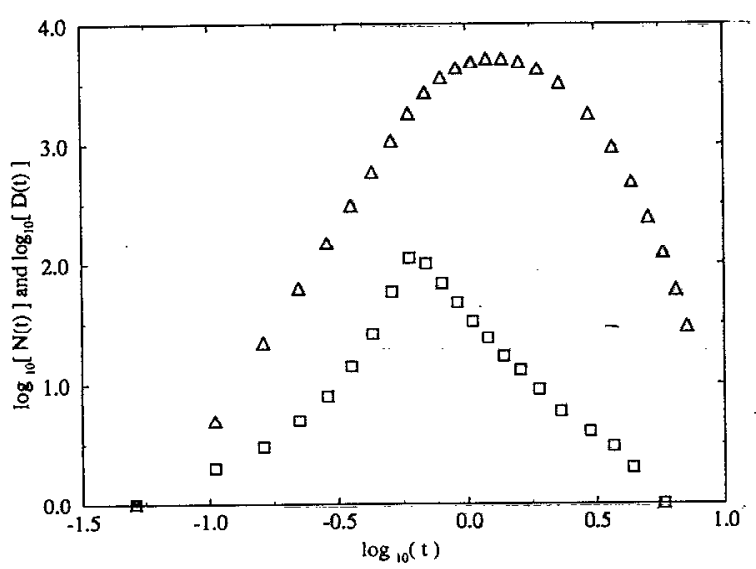

FIG. 1. The number of fragments $(\Delta)$ and diversity $(\square)$ as a function of time for an Euclidean square in a lattice with length $L=243$. The data refers to the corrosive rain model at $\Theta=\infty$.

$\bar{s}$ and $\bar{q}$ is, however, more complex. In actual numerical simulation we observe that $\bar{s}\left(t_{\sim r}\right) \simeq 2$, and $\bar{q}\left(t_{\mathscr{r}}\right) \simeq 1$, independent of the initial properties of the object. This has two consequences. First, the ratio, $\bar{q}\left(t_{\mathscr{N}}\right) / \bar{s}\left(t_{\mathscr{N}}\right)$, should be a constant independent of $M_{0}$ and $\bar{q}_{0}$. Consequently, one has in agreement with Eq. (1.3) that

$$
\mathscr{N} \sim M_{0} / \bar{q}_{0} \text {. }
$$

Secondly, using $\bar{q}\left(t_{\mathscr{r}}\right) \simeq 1$ in Eq. (3.8), at $t=t_{\mathscr{H}}$, we easily have the following relation:

$$
t_{\mathscr{H}}=\ln \bar{q}_{0} \text {. }
$$

From Eqs. (4.2) and (4.3) we realize that till this stage of fragmentation the process depends on the initial properties of the object, such as initial mass and average coordination.

In Figs. 2 and 3 we exhibit results for $\mathscr{N}$ vs $M_{0} / \bar{q}_{0}$ and $t_{\mathscr{r}}$ vs $\ln \bar{q}_{0}$ for a wide variety of objects. These plots are fitted, respectively, to $\mathscr{N}=a\left(M_{0} / \bar{q}_{0}\right)^{\alpha}$, and $t_{\mathscr{N}}=\epsilon \ln \bar{q}_{0}+e, \quad$ with $\quad a=0.50 \pm 0.02, \quad \alpha=1.00 \pm 0.01$, $\epsilon=1.00 \pm 0.05$, and $e=0.00 \pm 0.05$.

The result of numerical simulation in the present model shows that in the beginning of fragmentation diversity $D$ increases rapidly, reaches a maximum, and then decreases. At the maximum of diversity, $\mathscr{D}$, the number of fragments is still low compared to the maximum of fragment number, $\mathscr{N}$. At this point usually, one has on the average one fragment of each size except the very small ones where multiple fragments are possible. We could use this information to explain qualitatively the scaling property (1.4). Assuming that at $t=t_{\mathscr{P}}$ in the ideal case one has only one fragment of each type, the total mass is given by

$$
M\left(t_{\mathscr{D}}\right) \simeq 1+2+3+\cdots+\mathscr{P}=\mathscr{D}(\mathscr{D}+1) / 2 .
$$

Note in Fig. 1 that at the maximum of diversity, $\mathscr{D}, N(t)$ is larger than $\mathscr{D}$. This happens because there are usually several small mass fragments of each diversity. But for large systems this should only contribute to small corrections to Eq. (4.4). For large systems relation (4.4) is expected to hold and it simplifies to $M\left(t_{\mathscr{P}}\right) \simeq \mathscr{D}^{2} / 2$. Using this in Eq. (3.11) one arrives at the following relation:

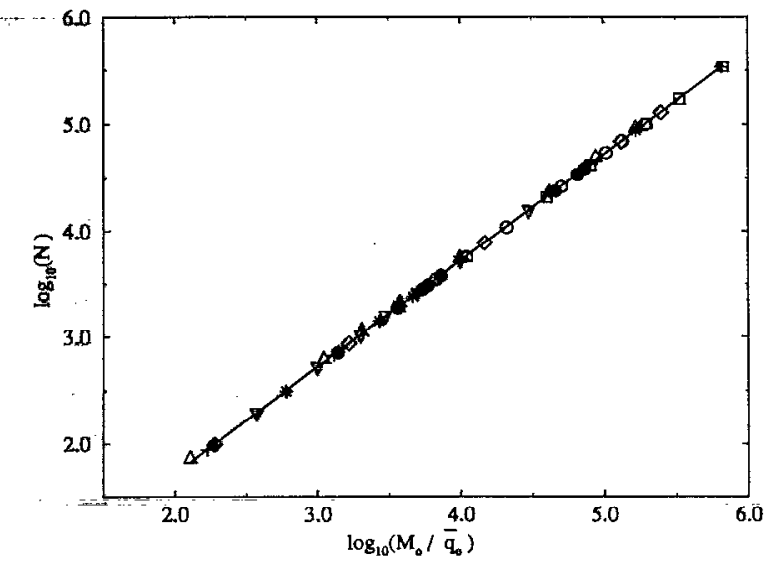

FIG. 2. $\log _{10} \mathscr{N}$ vs $\log _{10}\left(M_{0} / \bar{q}_{0}\right)$ for the corrosive rain model at $\theta=\infty$ for Euclidean objects on square lattices in space dimension $d=1(\nabla)$ of size $L=750,2000,4000,6000,10000,20000$, and $60000 ; d=2(\diamond)$ of size $L=27,81,150,200,243,729$, and $1000 ; d=3(*)$ of size $L=10,15$, $20,25,28,30,32,35$, and $100 ; d=4(O)$ of size $L=10,15,20,25,30$, 32 , and 35; and $d=5(\square)$ of size $L=8,10,13,15,18,20$, and 23; on triangular lattice $d=2(\Delta)$ of size $L=27,81,110,150,200,500,729$, and 1000; for Sierpinski carpet ( + ) with $L=27,81,243,729$, and 2187; for percolation cluster ( $)$ with $L=80,150,200$, and 729; for Menger sponge $(X)$ with $L=27$ and 81 . The straight line is fitted to $\mathscr{N} \sim\left(M_{0} / \vec{q}_{0}\right)^{\alpha}$ with $\alpha=1.00 \pm 0.01$.

$$
\mathscr{D}^{2} \sim \frac{M_{0}}{\bar{q}_{0}} \bar{q}\left(t_{\mathscr{D}}\right)
$$

On the scale of variation of $M_{0} / \bar{q}_{0}$, the quantity $\bar{q}\left(t_{\mathscr{D}}\right)$ could be considered a constant and thus one has

$$
\mathscr{D}^{2} \sim M_{0} / \bar{q}_{0} .
$$

Equations (4.2) and (4.6) lead to Eq. (1.4). In Fig. 4 we plot $\mathscr{N}$ vs $\mathscr{D}^{2}$. The results have been fitted to $\mathscr{N} \sim \mathscr{D}^{2 \beta}$, with $\beta=1.0 \pm 0.2$. With this plausible argumentation we have been able to identify the essential ingredients responsible for scaling (1.4).

In order to test the robustness of these scaling relations we also made numerical simulation for $\mathscr{N}$ and $\mathscr{D}$ for frag-

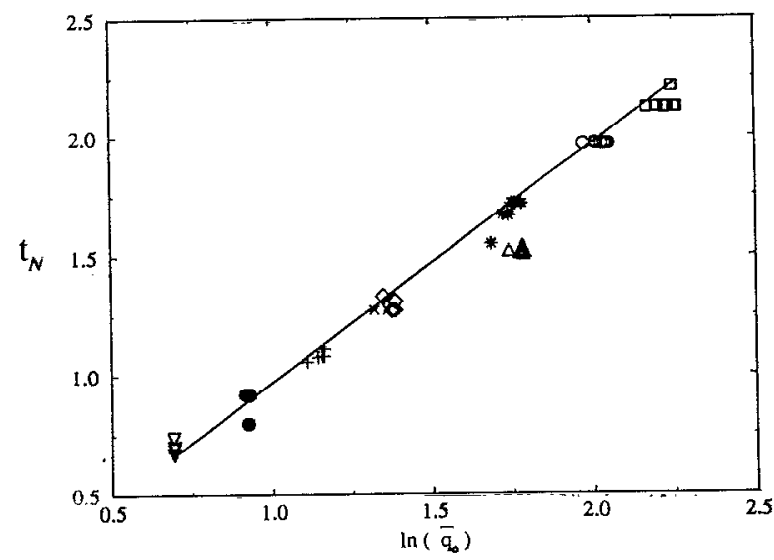

FIG. 3. $t_{t} \cdot$ vs $\log _{10}\left(\bar{q}_{0}\right)$ for all the objects of Fig. 2 for the corrosive rain model at $\Theta=\infty$. The straight line is fitted to $t, f=\epsilon \ln \bar{q}_{0}+e$ with $\epsilon=1.00$ \pm 0.05 and $e=0.00 \pm 0.05$. 


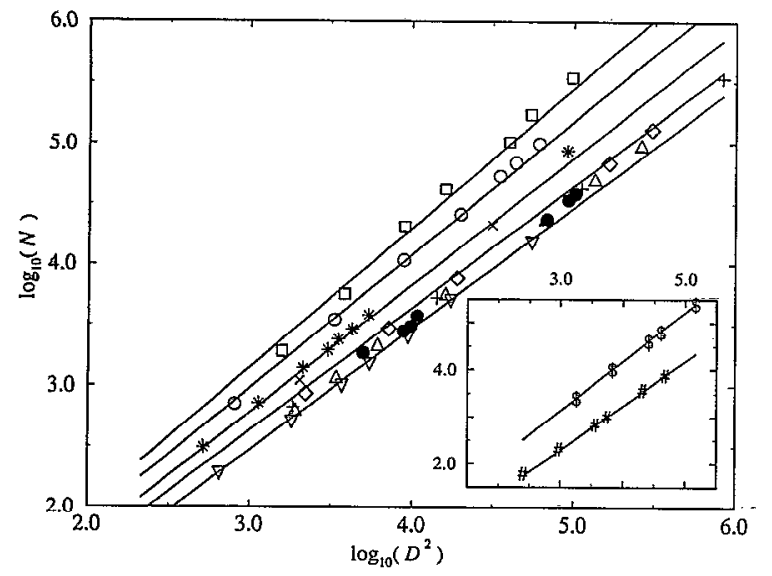

FIG. 4. $\log _{10}(\mathscr{N})$ vs $\log _{10}\left(\mathscr{D}^{2}\right)$ for all the objects of Fig. 2 for the corrosive rain model at $\Theta=\infty$. The inset displays the results for the random walk model (\$) and for the alternate lines model (\#), at $\theta=\infty$ for squares in space dimension $d=2$. All the straight lines are fitted to $\mathscr{N} \sim \mathscr{D}^{2 \beta}$ with $\beta=1.00 \pm 0.15$

mentation by random walk and by alternate lines presented before. In the case of the random walk model Eqs. (3.11) and (4.1) hold and also, the arguments leading to Eqs. (4.2) and (4.6). Consequently, scaling relations (1.3) and (1.4) should hold in this case. Results of simulations in these models are also shown in Fig. 4.

The case of fragmentation by alternate lines deserves special attention. In this model the mass is conserved, $M(t)=M_{0}$. The number of fragments increases monotonically till one has the desired label of fragmentation, namely, $\bar{s}\left(t_{\mathscr{N}}\right)=1$. As $M(t)=N(t) \bar{s}(t)$, one should have

$$
\mathscr{N} \sim M_{0}
$$

At the maximum of diversity, as before, it is plausible to assume there is one fragment of each possible size. Then the mass $M\left(t_{\mathscr{D}}\right) \equiv M_{0}$ could be written as

$$
M_{0} \simeq 1+2+3+\cdots+\mathscr{D}=\mathscr{D}(\mathscr{D}+1) / 2,
$$

which for large system simplifies to $M_{0} \sim \mathscr{D}^{2}$. Consequently, one establishes Eq. (1.4) in this case. We have verified scaling relations (4.7) and (4.8) via numerical simulation. We have exhibited in the inset of Fig. 4 the results of simulation with this modcl.

Next, we present results of numerical simulation on the time evolution of the system at infinite temperature. We have made prevision for time evolution of $M(t) / M_{0}$ [Eq. (3.5)] and $\bar{q}(t) / \bar{q}_{0}$ [Eq. (3.8)]. In Figs. 5(a) and 5(b) we plot the time evolution of these quantities which are fitted to $M(t) / M_{0}=r \exp (-\rho t)$, and $\bar{q}(t) / \bar{q}_{0}$ $=u \exp (-v t)$, respectively, where $r, u=1.00 \pm 0.03, \rho$, $v=1.00 \pm 0.02$. These results are in excellent agreement with the theoretical previsions: $r, u, \rho, v=1$. The results of numerical simulation in Fig. 5 are particularly relevant because the analytical rcsults are known in this case. This gives credibility to our numerical simulation, specially, in more complex situations where the analytic result is not known.

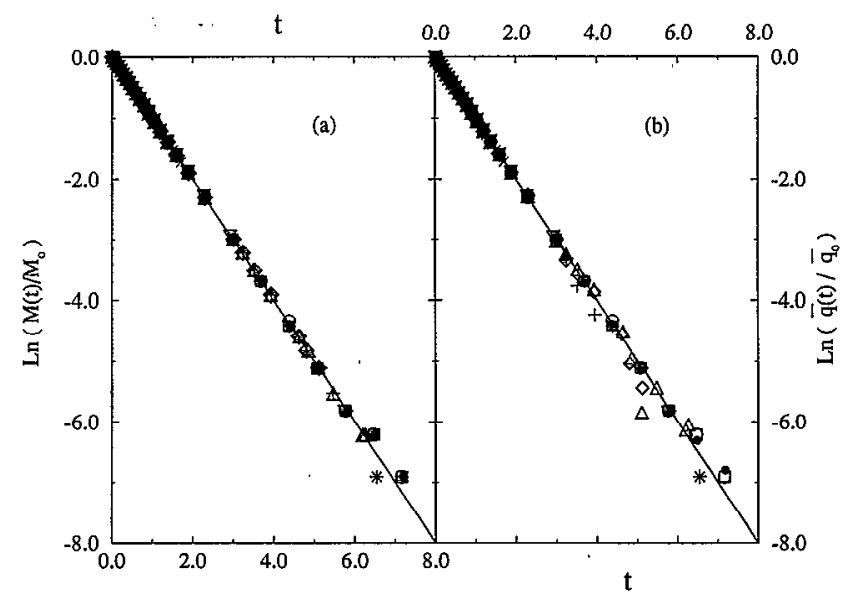

FIG. 5. (a) $\ln \left[M(t) / M_{0}\right]$ vs $t$, and (b) $\ln \left[\bar{q}(t) / \bar{q}_{0}\right]$ vs $t$ for all the objects of Fig. 2 for the corrosive rain model at $\Theta=\infty$. The straight lines are fitted to Eqs. (3.5) and (3.8), respectively.

From an analysis of the results of numerical simulation one finds the following scaling relation:

$$
\bar{s}(t)=\left(\frac{t}{\ln \bar{q}_{0}}\right)^{z}+1
$$

This is a generalization of the scaling relation $\bar{s}(t) \sim t^{z}$ considered previously. Note that in fragmentation $z$ is a negative exponent. This is consistent with the physical large $t$ limit, $\bar{s}(t \gg 1)=1$, and the result of simulation at $t=t_{\mathscr{T}}$, $s\left(t_{\mathscr{N}}\right)=2, t_{\mathscr{N}}=\ln \bar{q}_{0}$. Utilizing a log-log plot of $[\bar{s}(t)-1]$ vs $\left(t / \ln \bar{q}_{0}\right)$ the numerical value of the exponent $z$ is found for each type of object. These values are presented in Table I together with other exponents.

Now recalling the definition $N(t) \equiv M(t) / \bar{s}(t)$, and Eqs. (3.5) and (4.9), one encounters the following expression for $N(t)$ :

$$
N(t)=\left(M_{0} e^{-t}\right) /\left[1+\left(\frac{t}{\ln \bar{q}_{0}}\right)^{z}\right]
$$

where $z$ is the exponent listed in Table I. In the limiting conditions $t \ll 1, N(t) \sim t^{-z}(z<0)$, and $t \gg 1, N(t) \sim e^{-t}$.

In order to test the scaling relations (4.9) and (4.10) we have plotted in Fig. 6(a), $\bar{s}(t)$ vs $t$ and in Fig. 6(b) $N(t) /\left(M_{0} / \bar{q}_{0}\right)$ vs $t$ for a two-dimensional square of size

TABLE I. The numerical values of the exponents $z, \tau, w, \gamma$, for various objects in numerical simulation. The numerical accuracy of the exponents is about $10 \%$.

\begin{tabular}{lllllll}
\hline \hline \multicolumn{1}{c}{ Object } & $d$ & $\bar{q}_{0}$ & $z$ & $\tau$ & $w$ & $\gamma$ \\
\hline Line & 1 & 2.0 & -1.1 & 0.2 & 1.7 & 0 \\
Percolation cluster & 2 & 2.5 & -1.4 & 1.3 & 1.1 & 0 \\
Sierpinski carpet & 2 & 3.2 & -2.6 & 1.5 & 2.1 & 0.28 \\
Menger sponge & 3 & 3.7 & -2.9 & & & $\ldots$ \\
Square & 2 & 4.0 & -3.5 & 1.6 & 3.6 & 0.28 \\
Trapezoid & 2 & 6.0 & -4.2 & 1.5 & 4.2 & 0.28 \\
Cube & 3 & 6.0 & -4.4 & 1.9 & 4.5 & 0.28 \\
Hypercube & 4 & 8.0 & -5.2 & 2.1 & 6.5 & 0.28 \\
Hypercube & 5 & 10.0 & -5.8 & 2.3 & 7.0 & 0.28 \\
\hline \hline
\end{tabular}




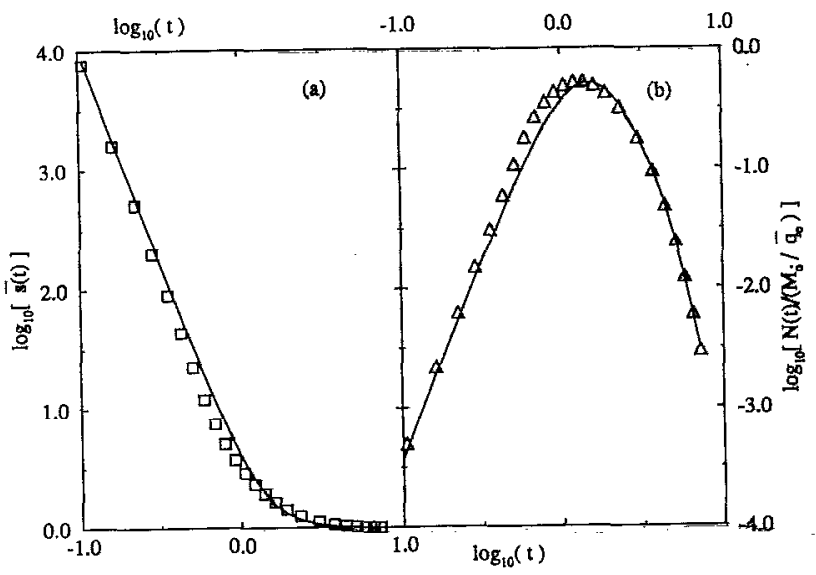

FIG. 6. (a) $\log _{10}[\bar{s}(t)]$ vs $\log _{10}(t)$ and (b) $\log _{10}\left[N(t) /\left(M_{0} / \bar{q}_{0}\right)\right]$ vs $t$ for a two-dimensional square of size $L=200$ for the corrosive rain model at $\theta=\infty$. The straight lines are fitted to Eqs. (4.9) and (4.10), respectively, with $z=-3.5$.

$L=200$. The full line in these cases are predictions of scaling relations (4.9) and (4.10) for $z=-3.5$ and agrees well with results of numerical simulations.

Now we would like to establish the scaling property (1.2) for the cluster size distribution, $n(s, t)$, from the present numerical study. The scaling property (1.1) has been established in detail for aggregation processes by diverse authors for large $t .{ }^{10}$ Qualitatively, fragmentation process can be considered as a time reversed version of aggregation. In aggregation for large $t$ one has a small number of fragments, whereas in fragmentation for small $t$ one has a small number of fragments. The $n(s, t)$ values in the small and large $t$ limits for fragmentation and aggregation, respectively, are supposed to be related by arguments of time reversal. Thus, for small $t$ the cluster size distribution for fragmentation processes is expected to obey scaling (1.1) with the sign of $w$ reversed. For small $t$, larger the $t$, larger is the number of fragments. This is reflected by a positive $w$ in Eq. (1.1). Thus one should expect intuitively the following scaling relation in case of fragmentation:

$$
n(s, t) \sim s^{-\tau} t^{w} f\left(s / t^{z}\right) .
$$

However, in our numerical simulation of fragmentation processes we found this to be true for average initial coordination of the object, $\bar{q}_{0}$, up to 3 . For higher $\bar{q}_{0}$, and for small $t$, the $t$ dependence of $n(s, t)$ is given, as in Eq. (1.2), by $t^{w s^{r}}$ rather than by $t^{w}$. Also, after an initial increase in the number of fragments, for large $t$ the number of fragments has to reduce in the process of fragmentation with consumption of mass, until all mass is consumed. This reduction is given by the exponential $e^{-\rho t}$ in Eq. (1.2). For small $t$ this exponential yields essentially a constant. The large $t$ behavior of $n(s, t)$, given by Eq. (1.2), bears some similarity with the "shattering" transition observed by various authors in fragmentation, where suddenly the rate of fragmentation is enhanced. ${ }^{19,20}$ In our fragmentation models, after the number of fragments, $N(t)$, has reached a

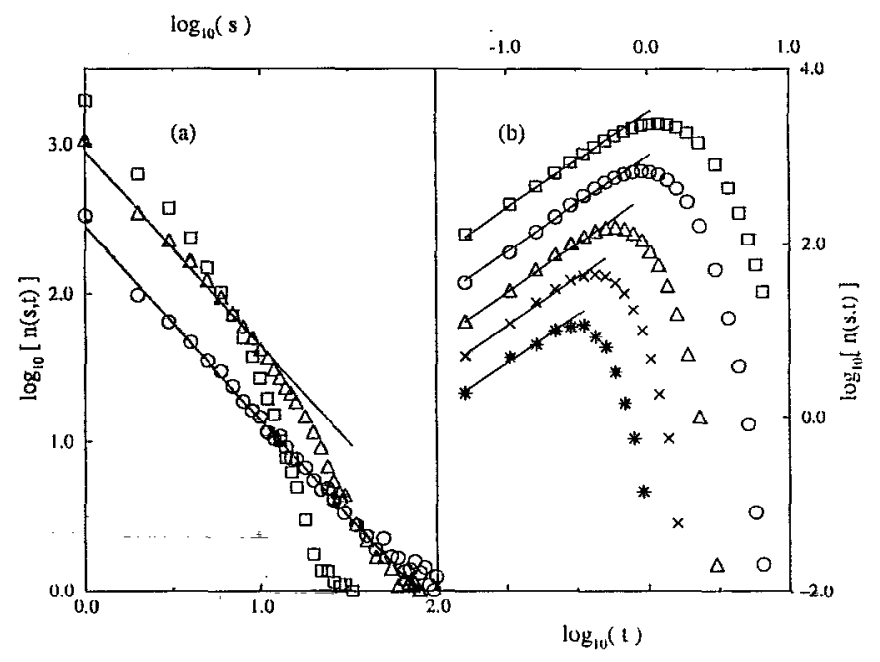

FIG. 7. (a) $\log _{10}[n(s, t)]$ vs $\log _{10}(s)$ with $t=0.10(O), 0.35(\Delta)$, and $0.70(\square)$, and (b) $\log _{10}[n(s, t)]$ vs $\log _{10}(t)$ with $s=1(\square), 2(O), 5(\Delta)$, $10(X)$, and $20(*)$, for a percolation cluster with $L=200$ for the corrosive rain model at $\Theta=\infty$. The straight lines are fitted to $n(s, t) \sim s^{-\tau}$ and $n(s, t) \sim t^{t}$, respectively, with $\tau=1.30 \pm 0.05$ and $w=1.10 \pm 0.05$.

maximum the rate of fragmentation increases rapidly and the whole mass is quickly consumed as can be seen in Fig. 1.

Next the results of numerical simulation of time evolution of cluster size distribution $n(s, t)$ are presented. It is to be noted that $n(s, t)$ is related to $s$ and $t$ via power law scaling in both aggregation [Eq. (1.1)] and fragmentation [Eq. (1.2)]. In Fig. 7 we present results of a percolation cluster with $L=200$. Figure 7 (a) presents $n(s, t)$ vs $s$ at times $t=0.10(O), 0.35(\triangle)$, and $0.70(\square)$, which corresponds, respectively, to $10 \%, 30 \%$, and $50 \%$ consumption of mass. We note in passing that the best power-law scaling relation for $n(s)$ is observed at the time $t_{\mathscr{D}}$ of maximum diversity irrespective the system [in Fig. $\left.7(\mathrm{a}), t_{\mathscr{D}}=0.1\right]$. Figure $7(\mathrm{~b})$ presents $n(s, t)$ vs $t$, for fixed $s=1(\square), 2(O)$, $5(\Delta), 10(X)$, and $20(*)$.

From Fig. $7(\mathrm{a})$ one can see that for $t \ll 1, n(s, t)$ has a power-law scaling in $s$ combined to a cutoff function for large s. From Fig. 7(b) one can see that for $t \ll 1, n(s, t)$ has a power-law scaling in $t$ combined to a exponential decay in $t$ for large $t$. These two types of scaling could be combined to yield

$$
n(s, t) \sim s^{-\tau} t^{w} e^{-t} f\left(s / t^{2}\right),
$$

where the cutoff function $f$ is defined after Eq. (1.1).

A relation between the exponents of Eq. (4.12) can be found from definition $M(t) \equiv \Sigma_{s} n(s, t) s$, which we rewrite in this case using Eq. (4.12) as

$$
M(t) \sim \int n(s, t) s d s \sim e^{-t} t^{w} \int s^{-\tau+1} f\left(s / t^{z}\right) d s .
$$

If one uses the transformation $x=s / t^{2}$ in Eq. (4.13), the following result is obtained:

$$
M(t) \sim e^{-t} t^{w-z(\tau-2)} .
$$




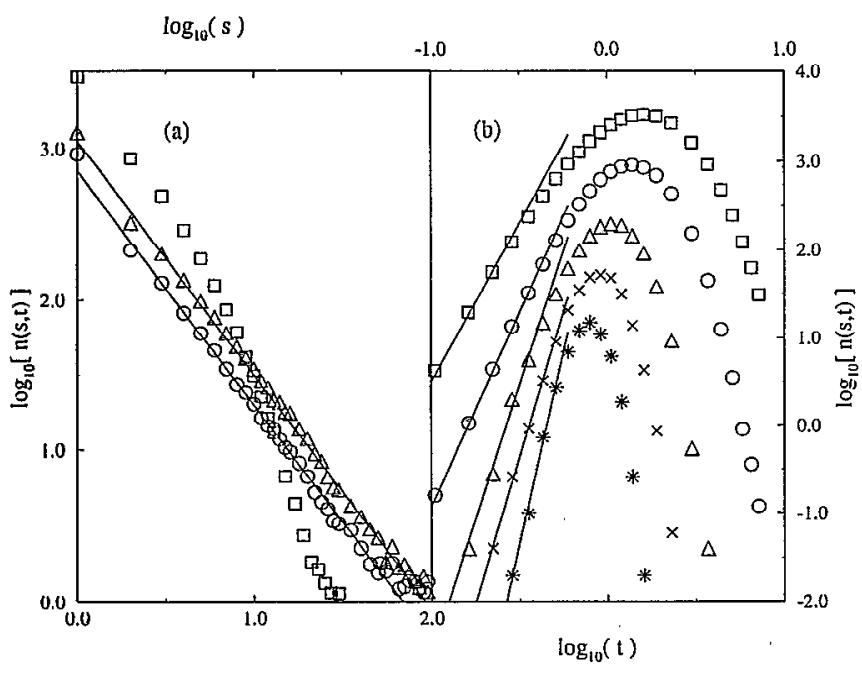

FIG. 8. (a) $\log _{10}[n(s, t)]$ vs $\log _{10}(s)$ with $t=0.10(0), 0.35(\Delta)$, and $0.70(\square)$, and (b) $\log _{10}[n(s, t)]$ vs $\log _{10}(t)$ with $s=1(\square), 2(O), 5(\triangle)$, $10(X)$, and $20(*)$, for a two-dimensional square with $L=200$ for the corrosive rain model at $\Theta=\infty$. The straight lines are fitted to $n(s, t) \sim s^{-\tau}$ and $n(s, t) \sim t^{g(s)}$, respectively, with $\tau=1.60 \pm 0.05$ and $g(s)=3.6,4.4,6.1$, 6.6 , and $8.7 \pm 0.1$.

Comparing Eqs. (3.5) and (4.14) one obtains the constraint $w=z(\tau-2)$. This relation was also obtained by Vicsek and Family for aggregation ${ }^{10}$ (which conserves mass). However, in their study the factor $e^{-t}$ was absent.

For all the objects utilized in numerical simulation in Fig. 2 we made plots similar to Fig. 7 and from such plots we found out the exponents $\tau$ and $w$. In these analyses we found that for objects with initial coordination $\bar{q}_{0}$ greater than a limiting initial coordination $q_{l} \simeq 3, n(s, t) \sim t^{g(s)}$, where $g$ is an increasing function of $s$. Gomes and Vasconcelos ${ }^{9}$ also observed a variation of $g$ with $s$ for Menger sponges and Sierpinski carpets (contrary to what they found for percolation clusters) with a completely different fragmentation dynamics. We conjecture that the existence of a size dependent exponent $g(s)$ is associated with an initial coordination $\bar{q}_{0}$ above the critical limit $\bar{q}_{0} \simeq 3$ and not with the particularities of the dynamics. To illustrate these facts we show in Figs. $8(a)$ and $8(b)\left(\bar{q}_{0} \simeq 4.00\right)$ the same plots of Fig. $7\left(\bar{q}_{0} \simeq 2.5\right)$ but now for squares with edge $L=200$. Although the dependence of $n$ with $s$ remains essentially the same $\left(n \sim s^{-1.30}\right.$ for percolation clusters [Fig. 7 (a)], $n \sim s^{-1.60}$ for squares [Fig. 8(a)]), the variation of $n$ with $t$ for squares is completely different since now $n$ scales with $t$ as $n \sim t^{g(s)}$ [Fig. 8(b)] and not as the percolation clusters where $n \sim t^{1.1}$ [Fig. 7(b)] irrespective the size $s$ of the fragments. For the sake of completeness we studied the behavior of all the objects with $\bar{q}_{0}>3$ employed in our numerical simulations. From a plot of $g(s)$ vs $s$, exhibited in Fig. 9 we find that $g(s)=w s^{\gamma}$, where $\gamma=0.28 \pm 0.03$ for all such objects. Actually, for $\bar{q}_{0} \leqslant 2.5$, $\gamma=0$, and one has the relation $w=(\tau-2) z$, and for $\bar{q}_{0} \geqslant 3.2$ $\gamma=0.28 \pm 0.03$, and the relation among the various exponents is not known. We find that the time dependence of $n(s, t)$ suffers a sudden modification at $\bar{q}_{0}=q_{l} \sim 3$, where $q_{l}$

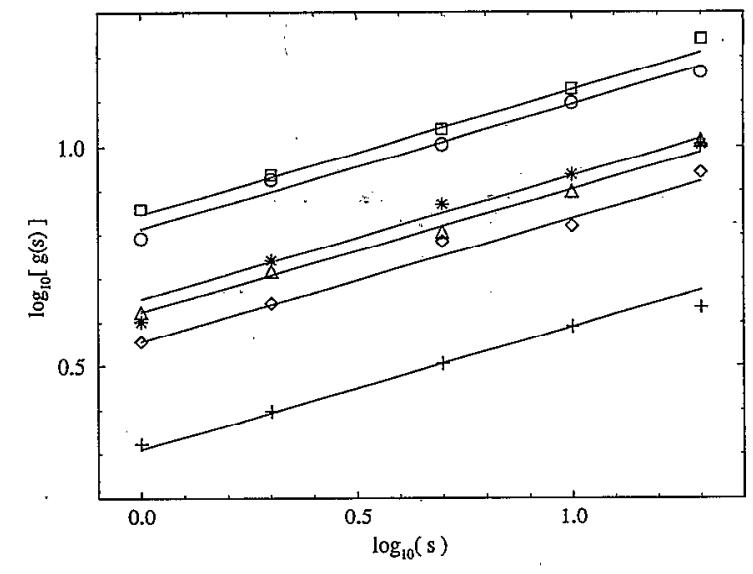

FIG. 9. $\log _{10}[g(s)]$ vs $\log _{10}(s)$ for compact objects on square lattice for $d=2(\diamond), d=3(*), d=4(\bigcirc), d=5(\square)$, on triangular lattice for $d=2$ $(\Delta)$ and for Sierpinski carpet $(+)$. The straight lines are fitted to $g(s)=w s^{\gamma}$ with $\gamma=0.28 \pm 0.03$ and $w$ given in Table I.

is termed limit coordination and is found to play an important role in the process of fragmentation.

In Table $I$ the values of the exponents for the different objects studied in the present article arc cxhibited. Analyzing these exponents, we see that the dynamic exponents $z$ and $w$ are mainly correlated with the limit coordination $q_{l}$ and secondarily with the space dimensionality $d$. The exponent $z$ varies logarithmically with $q_{l}$ as $z=3.00 \ln q_{l}-1.00$. On the contrary, the static exponent $\tau$ presents a strong correlation with $d$ and a weak correlation with $q_{l}$. The exponent $\tau$ varies logarithmically with $d$ as $\tau=0.90 \ln d+0.85$. The interval of variation of $\tau$ in Table I is quite restricted: $\tau=1.6 \pm 0.3$ for $d=2$ and 3 , and $2.5 \leqslant q_{l} \leqslant 6.0$. Turcotte ${ }^{15}$ has compiled experimental and theoretical data referring to 21 different fragmentation processes including chemical and nuclear explosions, interstellar grains and impacts of granular igneous rocks. The major part of their data is experimental and suggests that $\tau=1.2 \pm 0.2$. This overall estimate for the exponents $\tau$ is in agreement with the results of Table I ( $\tau=1.6 \pm 0.3)$.

We note that the exponent $\tau=1.9$ reported in Table I for a cube submitted to the fragmentation dynamics of the rain model studied here is also close to the values $\tau \simeq 0.7-$ 1.7 obtained with the maximum-entropy formalism ${ }^{17}$ in physical space. Also, the $\tau$ values for critical events is close to 2.2 , as in percolation in three dimensions around the critical point. This is also in agreement with our results. Howcver, a recent estimate, ${ }^{16} 2 \leqslant \tau \leqslant 4$, for nuclear fragmentation process is not consistent with present findings, possibly reflecting the difference in the dynamics of the fragmentation processes.

It is also interesting to observe that cluster cluster aggregation (CCA) studied by Vicsek and Family ${ }^{10}$ in $d=2$ presents $z=1.4 \pm 0.2, w=1.7 \pm 0.2, \quad \tau=0.75 \pm 0.15$, and $\bar{q}_{0} \simeq 2$. The exponents $z, \tau$ and $w$ for CCA are close to those shown in Table I for the percolation cluster which has $\bar{q}_{0}=2.5$ in $d=2$. 


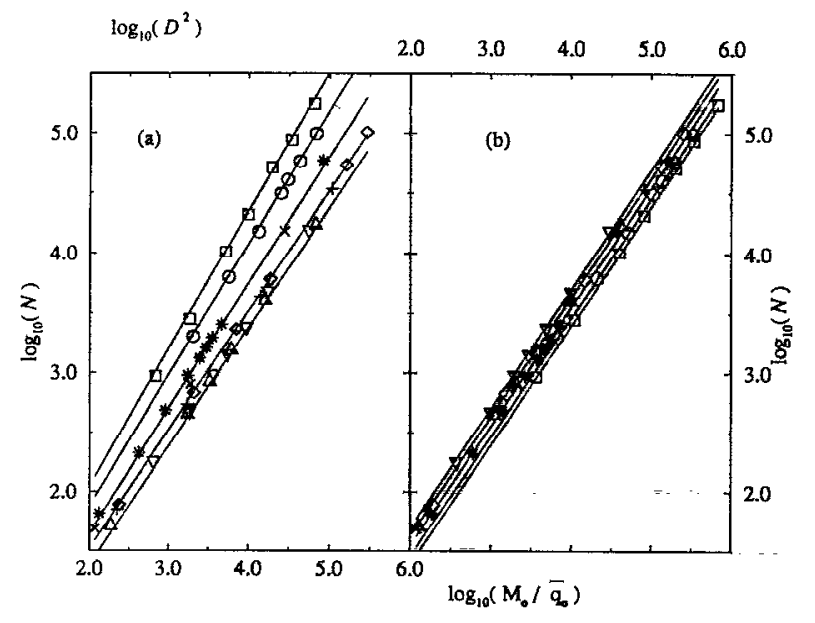

FIG. 10. (a) $\log _{10}(\mathscr{N})$ vs $\log _{10}\left(\mathscr{D}^{2}\right)$, and (b) $\log _{10}(\mathscr{N})$ vs $\log _{10}\left(M_{0} / \bar{q}_{0}\right)$ for all the objects of Fig. 2 for the corrosive rain model at $\Theta=5$. The straight lines are fitted to $\mathscr{N}=b(d, \Theta) \mathscr{D}^{2 \beta}$ and $\mathscr{N}=a(d, \Theta)$ $\left(M_{0} / \bar{q}_{0}\right)^{\alpha}$, respectively, with $\alpha=1.00 \pm 0.01$ and $\beta=1.0 \pm 0.1$.

\section{B. Kinetic domain: Finite temperature}

Next we consider the more realistic reactivity $p_{i}=\exp \left(-q_{i} / \Theta\right)$, where $q_{i}$ is the coordination of site $i$, and $\Theta$ is the reduced temperature. In numerical simulation we used two temperatures $\Theta=5$ and 1 . The time evolution of $N(t)$ and $D(t)$ are similar to the infinite temperature case. From a critical study of the maxima $\left(t_{\mathcal{H}}, \mathcal{N}\right)$ and $\left(t_{\mathscr{D}}, \mathscr{D}\right)$, we find that $\mathscr{N}$ decreases with decreasing temperature $\Theta$, and $\mathscr{D}$ remains constant. However, the scaling relations (1.3) and (1.4) remain unchanged. In Figs. 10 (a) and 10 (b) we plot $\mathscr{N}$ vs $\left(M_{0} / \bar{q}_{0}\right)$ and $\mathscr{N}$ vs $\mathscr{D}^{2}$, respectively, for all the objects in Fig. 2 at temperature $\Theta=5$. The lines are fitted to $\mathscr{N} \sim\left(M_{0} / \bar{q}_{0}\right)^{\alpha}$ and $\mathscr{N} \sim \mathscr{D}^{2 \beta}$, respectively, with $\alpha=1.00 \pm 0.01$ and $\beta=1.0 \pm 0.1$.

The times to reach the maxima $t_{\mathcal{H}}$ and $t_{\mathscr{D}}$ in the kinetic domain vary linearly with $\bar{q}_{0}$, in contrast to the infinite temperature limit where this dependence is logarithmic. As a result of numerical simulation at $\Theta=5$ we find the numerical relations $t_{\mathscr{r}}=0.68 \bar{q}_{0}-0.55$ and $t_{\mathscr{D}}=0.80 \bar{q}_{0}-1.60$. For $\Theta=1$, we also observed similar linear relations.

We observe that the time evolution of both mass $M(t)$ and average coordination $\bar{q}(t)$ are quite different from those in the infinite temperature limit. From a result of numerical simulation we find that for $t \gg 1$, these time evolutions at finite temperatures are given by

$$
M(t) \sim M_{0} e^{-\rho t}
$$

and

$$
\bar{q}(t) \sim \bar{q}_{0} e^{-v t},
$$

to be compared with Eqs. (3.8) and (3.5), respectively, in the infinite temperature case. For small times the dependence is more complicated. In the finite temperature case both $\rho$ and $v$ are found to be positive constants less than

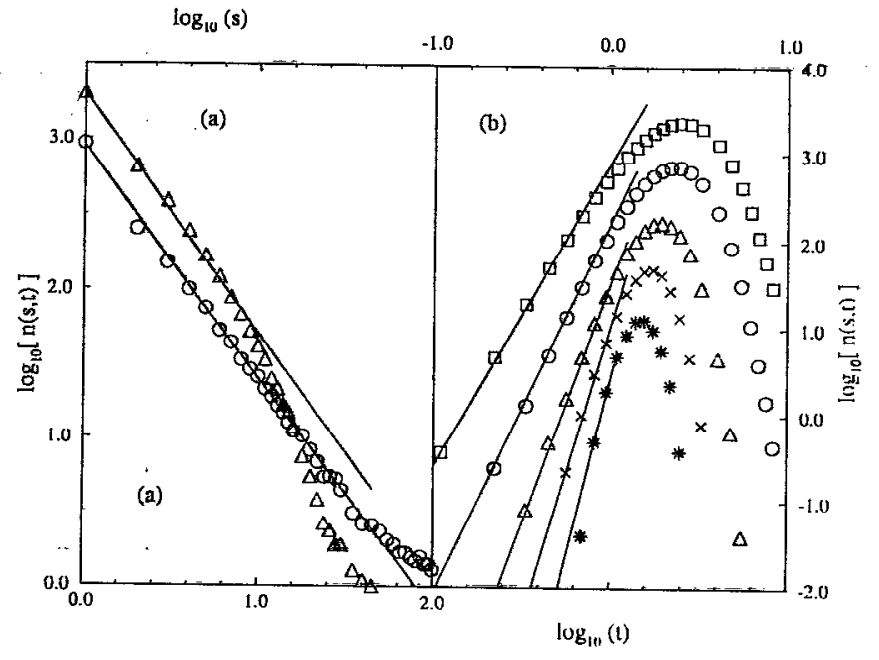

FIG. 11. (a) $\log _{10}[n(s, t)]$ vs $\log _{10}(s)$ for $t=0.95(O)$ and $1.65(\Delta)$ and (b) $\log _{10}[n(s, t)]$ vs $\log _{10}(t)$ for $s=1(\square), 2(O), 5(\Delta), 10(X)$, and 20 (*) for a two-dimensional square with $L=200$ for the corrosive rain model at $\Theta=5$. The straight lines are fitted to $n(s, t) \sim s^{-\tau}$ and $n(s, t)$ $\sim t^{m s^{\gamma}}$, respectively, with $\tau=1.6, w=3.5$, and $\gamma=0.29$.

unity, independent of the initial properties of the object concerned, and a function of the reduced temperature, $\theta$, only.

For the average fragment size $\bar{s}(t)$, we find for $t \ll 1$, $\bar{s}(t) \sim t^{z}(z<0)$, where $z$ is the same exponent of Table $\mathrm{I}$. For the total number of fragments $N(t)$, for $t \ll 1$ one has the same time dependence as in the infinite temperature case: $N(t) \sim t^{-z}$. However, for $t \gg 1, N(t)[\equiv M(t) / \bar{s}(t)]$ suffers the same change as mass $M(t)$ above, and one encounters $N(t) \sim \exp (-\rho t)$.

For the cluster size distribution, $n(s, t)$, in the kinetic domain we find that the scaling relation (1.2) is valid at a finite temperature, where the exponents $\tau, w, \gamma, z$, and the cutoff function $f$ were found to be the same as in the infinite temperature case. For all the objects utilized in Fig. 2, we made plots similar to Figs. 7, 8, and 9. To illustrate we show in Figs. 11(a) and 11(b) the results for $n(s, t)$ vs $s$ and $t$, respectively, at $\Theta=5$, for a two-dimensional square of size $L=200$. In Fig. 11 (a) we present results of numerical simulation at $t=0.95(O)$, and $1.65(\triangle)$. These correspond to $50 \%$ and $70 \%$ of mass consumption, respectively. We note here that the best power-law scaling relation for $n(s)$ is obtained at the time $t_{\mathscr{D}}$ of maximum diversity irrespectively of the system [in Fig. 11(a), $\left.t_{\mathscr{D}}=0.95\right]$, as in Fig. 7(a). If we plot $n(s)$ vs $s$ for $t$ near $t_{\mathscr{D}}$, we find fits as good as in Fig. 8(a) where $O$ represents $t=t_{\mathscr{D}}$ and $\Delta$ represents $t$ near $t_{\mathscr{D}}$. The straight lines represent the analytical scaling relations $n(s, t) \sim s^{-\tau}$ with $\tau=1$.6. In Fig. 11(b) we present results of the numerical simulation for $s=1(\square), 2(O), 5(\triangle), 10(\times)$, and 20 (*). The straight lines represent $n(s, t) \sim t^{g(s)}$. In order to analyze the function $g(s)$, we plotted $g(s)$ vs $s$ as in Fig. 9 and we found the scaling relations $g(s)=w s^{\gamma}$ with $w=3.5$ and $\gamma=0.29$.

It is interesting to note that the exponents $w, \tau, z$, and 
$\gamma$ did not change with the introduction of temperature and are essentially the same as in Table I for all the objects. This fact suggests that these exponents are determined by the statistical nature of the process and not by any physical properties. Consequently, we believe that these exponents will be the same as in actual physical fragmentation process by corrosive rain. This expectation is further strengthened by the numerical simulation of Gomes and Vasconcelos ${ }^{9}$ who found essentially the same values for these exponents, wherever they calculated these exponents, in a different model of attack for fragmentation.

\section{SUMMARY AND CONCLUSIONS}

To our knowledge the present work is the first attempt to investigate systematically a fragmentation model in a wide range of space dimension $(1 \leqslant d \leqslant 5)$ and initial coordination $\left(2 \leqslant \bar{q}_{0} \leqslant 10\right)$. We simulated an acid rain attack to a surface by a corrosive rain model and we studied some quantities involved in this process such as mass $M(t)$, number of fragments $N(t)$, diversity of fragments $D(t)$, mean size of fragments $\bar{s}(t)$, mean coordination of system $\bar{q}(t)$ and the number of fragments of size $s n(s, t)$. We developed analytic rate equations for the time evolution of mass and coordination of the system. We made also extensive numerical simulation of the process.

We studied the random domain of this model (infinite temperature) in which the rate of corrosion is determined only by the randomness in choosing a site. In this special case the equations allow analytic solution that were in excellent agreement with the results of numerical simulation. We have confirmed in our study two scaling relations $\mathscr{N} \sim \mathscr{D}^{2}$ that related the maximum of fragments $\mathscr{N}$ with the maximum of diversity $\mathscr{D}$, and $n(s, t)$ $\sim s^{-\tau} t^{w s^{\gamma}} e^{-\rho t} f\left(s / t^{z}\right)$, where $\tau, w, \gamma$, and $z$ are important exponents to describe the process (Table I), $\rho$ is a constant that controls the exponential consumption of mass and $f(x)$ is a cutoff function.

Two other completely different models of fragmentation were also considered. These were fragmentation by random walk and by alternate horizontal and vertical lines. In both cases the scaling relation $\mathscr{N} \sim \mathscr{D}^{2}$ holds wcll. From the robustness of this relation with respect to variation of attack, dimension, and topology of the object, this expression is found to play an equivalent role as the equations of state in equilibrium processes.

In order to test the generality of the exponents $\tau, w, \gamma$, and $z$, we made modifications in the model of fragmentation, and considered fragmentation by corrosive rain in the kinetic domain at finite temperature. In this model the probability of successful attack on a site depends on its binding to the object. This model of attack depends on the physical properties of the system. However, the numerical values of the exponents remain unchanged. Hence these exponents are expected to remain unchanged in a wide class of fragmentation processes.

Comparing the exponents obtained in our simulations $\tau, w, \gamma$, and $z$ with the experimental exponents obtained by Turcotte, ${ }^{15}$ and the theoretical exponents obtained by Vicsek and Family ${ }^{10}$ and by Gomes and Vasconcelos, ${ }^{9}$ we noted that although they utilize different dynamics, the exponents are in good agreement with ours. These results lead us to conclude that critical exponents associated to different dynamics generating cluster distributions depend heavily on $\bar{q}_{0}$ and $d$ and not on the intricacies of a particular dynamics. Indeed an examination of Table I shows that systems with a same value of $d$ present completely different sets of critical exponents for different values of $\bar{q}_{0}$, , i.e., the space dimension is not sufficient to specify the critical exponents and at least two "good" numbers, $\bar{q}_{0}$ and $d$, possibly fix the critical exponents. This situation is different if compared with equilibrium phenomena where the space dimension fix the values of the critical exponents.

\section{ACKNOWLEDGMENT}

The work is supported in part by the Conselho Nacional de Desenvolvimento Científico e Tecnológico ( $\mathrm{CNPq}$ ) of Brazil.

${ }^{1}$ J. J. Gilvarry, J. Appl. Phys. 32, 391 (1961).

${ }^{2}$ B. L. Holian and D. E. Grady, Phys. Rev. Lett. 60, 1355 (1988)

${ }^{3}$ A. R. Kerstein and A. L. R. Bug, Phys. Rev. B 34, 1754 (1986).

${ }^{4}$ E. W. Montroll and R. Simha, J. Chem. Phys. 8, 721 (1940); F. Family, P. Meakin, and J. M. Deutsch, Phys. Rev. Lett. 57, 774 (1986).

${ }^{5}$ X. Campi, J. Phys. A 19, L-917 (1986); A. Z. Mekjian, Phys. Rev. Lett. 64, 2125 (1990).

${ }^{6}$ D. E. Grady and M. E. Kipp, J. Appl. Phys. 58, 1210 (1985).

${ }^{7}$ B. F. Edwards, M. Cai, and H. Han, Phys. Rev. A 41, 5755 (1990).

${ }^{8} \mathrm{M}$. Sahimi and T. T. Tsotsis, Phys. Rev. Lett. 59, 888 (1987); M. Sahimi, Phys. Rev. A 43, 5367 (1991).

${ }^{9}$ M. A. F. Gomes and G. L. Vasconcelos, J. Phys. A 22, L-757 (1989); Comput. Phys. Commun. 54, 257 (1989); M. A. F. Gomes, G. L. Vasconcelos, and S. L. Meira, Quim. Nova 11, 133 (1988).

${ }^{10}$ T. Vicsek and F. Family, Phys. Rev. Lett. 52, 1669 (1984); T. Vicsek, P. Meakin, and F. Family, Phys. Rev. A 32, 1122 (1985).

${ }^{11}$ A. Meesters and M. H. Ernst, J. Colloid Interface Sci. 119, 576 (1987); C. M. Sorensen, H. X. Zhang, and T. W. Taylor, Phys. Rev. Lett. 59, 363 (1987); R. D. Vigil and R. M. Ziff, ibid. 61, 1431 (1988).

${ }^{12}$ Z. Jaeger and R. Englman, J. Appl. Phys. 59, 4048 (1986).

${ }^{13}$ X. Z. Hu, B. Cotterell, and Y. W. Mai, Philos. Mag. Lett. 57, 69 (1988).

${ }^{14}$ J. Klafter and M. F. Shlesinger, Proc. Natl. Acad. Sci. (USA) 83, 848 (1986).

${ }^{15}$ D. L. Turcotte, J. Geophys. Res. 91, 1921 (1986).

${ }^{16}$ ALADIN Collaboration, GST Report No. GST-92-23.

${ }^{17}$ R. Englman, N. Rivier, and Z. Jaeger, Philos. Mag. B 56, 751 (1987).

${ }^{18}$ N. F. Mott and E. H. Linfoot, Ministry of Supply AC 3348, January, 1943; N. F. Mott, ibid. AC 3642, March, 1943; AC 4035, May 1943.

${ }^{19}$ E. D. McGrady and R. M. Ziff, Phys. Rev. Lett. 58, 892 (1987); J. Phys. A 18, 3027 (1985).

${ }^{20}$ Z. Cheng and S. Redner, Phys. Rev. Lett. 60, 2450 (1988); R. Botet and M. Ploszajczak, ibid. 69, 3696 (1992); B. F. Edwards, M. Cai, and H. Han, Phys. Rev. A 41, 5755 (1990). 